\title{
Answering user queries from hotel ontology for decision making
}

\begin{abstract}
Semantic web comes out with the vision of making human readable information to be machine processable. Ontology, the core of semantic web, with concept instantiations serves as a domain knowledge base while semantic web query language provides retrieval of that information. In this paper, we presented a system that populates hotel related information in the ontology and a natural language querying platform to retrieve the information from a common interface for decision making. A simple user experiment shows that the system is time effective and helpful in making decisions with minimum queries as compared to browsing even with selected sites.
\end{abstract}

Keyword: Ontology; Query answering; Hotel search; Natural language queries; SPARQL 